\title{
DESIGN, SYNTHESIS, MOLECULAR DOCKING, AND BIOLOGICAL EVALUATION OF PYRAZOLE 1-CARBOTHIAMIDE INCORPORATED ISOXAZOLE DERIVATIVES
}

\author{
RADHIKA TUMMA ${ }^{1,2}$, HARINADHA BABU VAMARAJU ${ }^{1 *}$ \\ ${ }^{1}$ Department of Pharmaceutical Chemistry, G. Pulla Reddy College of Pharmacy, Hyderabad, Telangana, India. ${ }^{2}$ Department of Pharmacy, \\ University College of Technology, Osmania University, Hyderabad, Telangana, India. Email: radhikavanam25@gmail.com
}

Received: 14 February 2018, Revised and Accepted: 09 April 2019

\section{ABSTRACT}

Objectives: Novel isoxazole incorporated pyrazole carbothiamide 5 (a-r) was designed and synthesized, docked and evaluated for anticancer activity Michigan Cancer Foundation-7 (MCF-7), and breast cancer cell lines.

Materials and Methods: Designed compounds were synthesized by the condensation of 1-(5-methyl-3-(4-nitrophenyl) isoxazole-4-yl) -3-(substitutedphenyl) prop-2-en-1-one (4) with thiosemicarbazides and substituted thiosemicarbazides to give the target molecules 5 (a-r). To predict the affinity and activity of the ligand molecule, the docking program Accelrys Discovery Studio 2.1 was employed to generate different bioactive binding poses of designing molecules at the active site of human Dihydrofolate Reductase (DHFR) (PDB ID: 1KMS). All the synthesized compounds were characterized based on the spectral and elemental analysis data. Antiproliferative activity was performed against MCF-7 breast cancer cell lines.

Results: All the synthesized compounds showed the characteristic peaks in Fourier-transform infrared, ${ }^{1} \mathrm{H} \mathrm{C}{ }^{13} \mathrm{NMR}$, and mass spectral analysis. During docking, all the synthesized compounds 5 (a-r) exhibited higher fitness scores with minimum three bonding interaction with the active site human DHFR (PDB ID: 1KMS). In the MTT assay based on MCF-7 breast cancer cell lines, most of the compounds exhibited significant activity. In the antiproliferative assay against MCF-7 cell lines, most of the compounds exhibited potent activity with IC $_{50}$ values in micromolar concentrations. Compounds $\mathbf{5 a}, \mathbf{5 b}, \mathbf{5 f}, \mathbf{5 h}$, and $\mathbf{5 k}$ have exhibited significant anticancer activity.

Conclusions: The derivatives were synthesized in quantitative yields. New derivatives possess the antiproliferative activity.

Keywords: Isoxazole, Pyrazole, Carbothiamide, Antiproliferative activity.

(c) 2019 The Authors. Published by Innovare Academic Sciences Pvt Ltd. This is an open access article under the CC BY license (http://creativecommons. org/licenses/by/4. 0/) DOI: http://dx.doi.org/10.22159/ajpcr.2019.v12i5.32591

\section{INTRODUCTION}

Cancer [1] is projected as one of the second most prevalent types of all diseases responsible for mortality overall the world. Interrupting the folate metabolism in cancerous cells may accommodate a favorable chance in cancer chemotherapy as a consequence of the inhibition of the biosynthesis of nucleic acid precursors. Thus, an inhibitor of the DHFR enzyme in cancerous cells would result in the inhibition of tetrahydrofolate synthesis; consequently, the nucleic acid precursors [2-4]. Isoxazoles and pyrazoles were used widely for the pharma world. Pyrazoles are used as anti-inflammatory [5], antioxidant [6], anticonvulsant [7], anticancer [8], antimicrobial [9,10], antiviral [11], and wide range of activities [12]. On the other hand, isoxazoles possess an extensive lineup of biological activities as well as forming an integrate of varied biodynamic agents $[13,14]$. A number of isoxazoles and related compounds are known to exhibit antitumor [15], anti-HIV [16], anti-inflammatory [17], antioxidant [18], antiviral [19], and antimicrobial activities [20]. Various biological activities of pyrazoles and isoxazole pharmacophores led to the search for new bioactive compounds of this class [21]. In the present study, while designing target molecules, isoxazole heterocycle is condensed with thiosemicarbazide to obtain pyrazole-1-carbothiamide incorporated isoxazole scaffold to obtain new hybrid molecules. Further, the designed molecules were computationally docked into target human DHFR (PDB ID: 1KMS) using an Accelrys discovery studio to gain some structural insights into the binding mode of designing molecules. The compounds that demonstrated a high fitness score in comparison with the reference drug, doxorubicin and are further planned to screen anti-proliferation study against Michigan Cancer Foundation-7 (MCF-7) cell lines and antitubercular activity.

\section{METHODS}

\section{Chemistry}

All the chemicals and solvents used were of synthetic grade from SD Fine Chemicals Ltd., (Mumbai, India), and Avra Chemicals Pvt. Ltd., Hyderabad. Completion of the reactions was monitored by analytical thin-layer chromatography (TLC) using E-Merck $0.25 \mathrm{~mm}$ silica gel plates. Visualization was accomplished with ultraviolet light $(256 \mathrm{~nm})$ and iodine chamber. Synthesized compounds were purified by a recrystallization process. The purity of the compounds was checked by a single spot in TLC and a solvent system for TLC was determined on trial and error basis. Melting points were determined in open capillary tubes using ANALAB melting point apparatus and were uncorrected. All the ${ }^{1} \mathrm{H}$ NMR spectra were recorded on Varian $400 \mathrm{MHz}$ spectrometer using $\mathrm{CDCl}_{3}$ as solvent and tetramethyl silane as an internal standard. Chemical shift values are listed in $\delta$ scale. The Fourier-transform infrared (FT-IR) spectra were recorded on Shimadzu FT-IR spectrophotometer using $1 \%$ potassium bromide discs. Mass spectra of the compounds were recorded on electronic ionization mass spectra on Agilent 1100 series.

\section{Synthesis of 4-nitrobenzaldehyde oxime (1)}

4-nitrobenzaldehyde $(0.01 \mathrm{mmol})$ was placed in a RBF containing $10 \mathrm{ml}$ of ethanol. To the above mixture hydroxylamine hydrochloride $(0.02 \mathrm{mmol})$ and anhydrous sodium acetate $(0.02 \mathrm{mmol})$ were added with constant stirring and reflux for 3-4 $\mathrm{h}$. The reaction was monitored by TLC. The resulting solution was poured into crushed ice. The obtained precipitate was filtered and thoroughly washed with water and air dried. TLC solvent systems - pet. ether:ethyl acetate (70:30). 


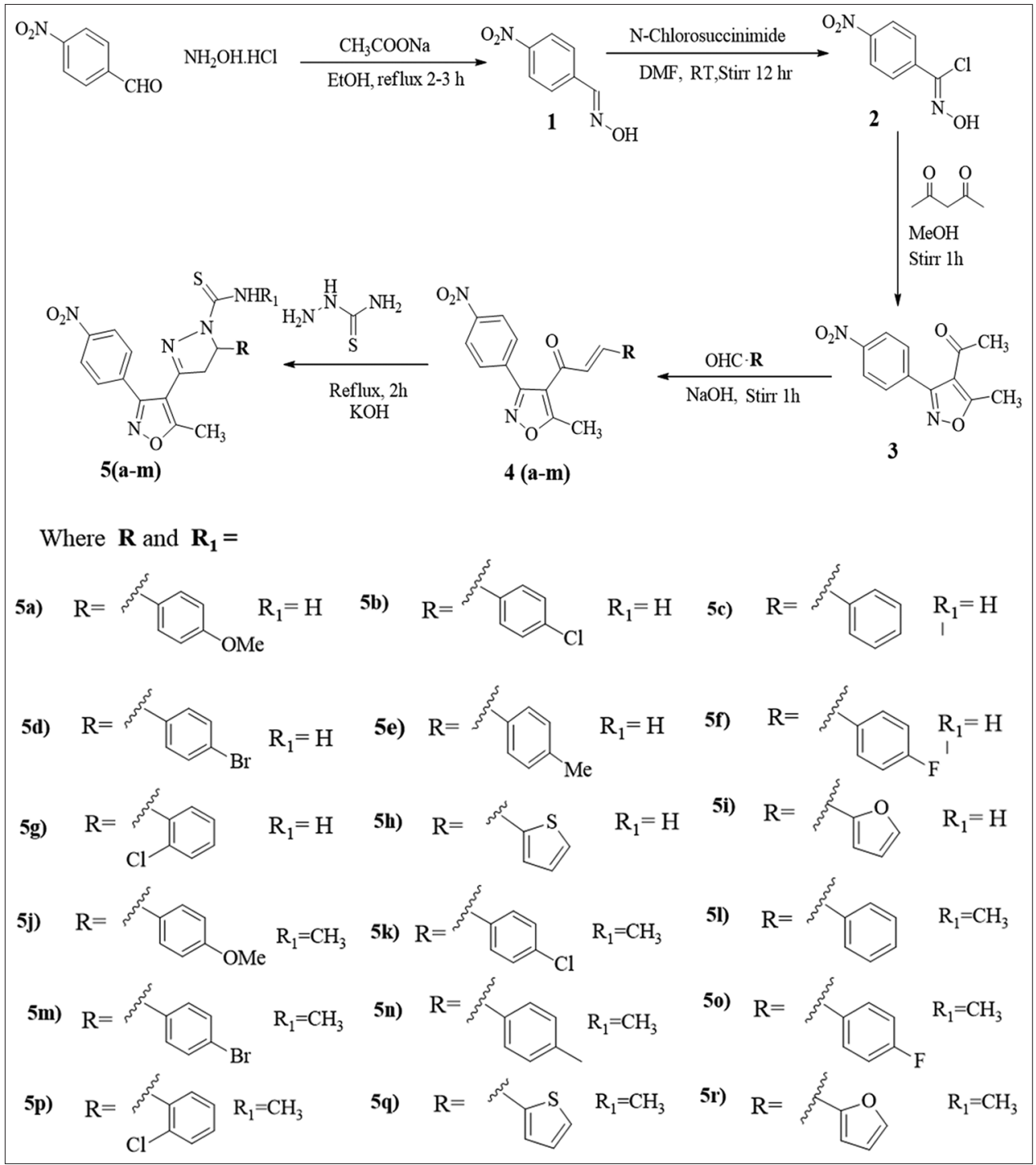

Fig. 1: Scheme for the synthesis of 5-(4-Substitutedphenyl)-3-(5-methyl-3-(4-nitrophenyl) isoxazole-4-yl)-4,5-dihydro-1H-pyrazole-1carbothioamide/ $\mathrm{N}-1$-carbothiamides

Synthesis of $N$-hydroxy-4-nitrobenzimidoyl chloride (2)

4-Nitrobenzaldehyde oxime $(0.01 \mathrm{mmol})$ and $\mathrm{N}$-Chlorosuccinamide $(0.01 \mathrm{mmol})$ were dissolved in dimethylformamide then it was stirred for overnight at room temperature. The reaction was monitored by TLC. After completion of the reaction, solution was poured into ice cold water. The obtained precipitate was filtered and washed thoroughly with ice cold water and air dried. TLC solvent systems - pet. ether:ethyl acetate $(70: 30)$

Synthesis of 1-(5-methyl-3-(4-nitrophenyl) isoxazol-4-yl) ethanone (3)

To the sodium hydroxide $(0.02 \mathrm{~mol})$ in methanol, acetylacetone $\left(0.04 \mathrm{mmol}\right.$ ) was added and maintained at $0-5^{\circ} \mathrm{C}$ and stirred for $10-15 \mathrm{~min}$ by maintaining $\mathrm{pH} 10$. To this mixture $0.02 \mathrm{mmol}$, aryl oxime was added and stirred on a magnetic stirrer for $1-2 \mathrm{~h}$. The completion of the reaction was monitored by TLC. After the completion of the reaction, the reaction mixture was poured into crushed ice. The precipitate was filtered and dried in air. TLC solvent systems - pet. ether:ethyl acetate (70:30).

Synthesis of 1-(5-methyl-3-(4-nitrophenyl) isoxazol-4-yl) -3-(substituted phenyl) prop-2-en-1-one (4)

An equimolar quantity of isoxazole ketone $(0.01 \mathrm{mmol})$ and substituted aryl aldehyde $(0.01 \mathrm{mmol})$ were dissolved in $15-20 \mathrm{ml}$ of alcohol in it $\mathrm{NaOH}(10 \%)$ added and stirred on magnetic stirrer over a period of $30 \mathrm{~min}$ to $1 \mathrm{~h}$. The reaction was monitored by TLC. The formed solid was filtered, washed with alcohol and dried in air. TLC solvent systems - pet. ether:ethyl acetate (70:30).

Synthesis of 5-(substituted phenyl) -3-(5-methyl-3-(4-nitrophenyl) isoxazol-4-yl) -4,5-dihydro-1H-pyrazole-1-carbothioamide and 5-(substituted phenyl) - $N$-methyl-3-(5-methyl-3-(4-nitrophenyl) isoxazol-4-yl) -4,5-dihydro-1H-pyrazole-1-carbothioamide5 (a-r) The intermediate 4 (a-m) (0.001 mol), thiosemicarbazide and substituted thiosemicarbazide $(0.001 \mathrm{mmol})$ were taken into R.B.F containing ethanol to it, $\mathrm{KOH}(10 \%)$ solution was added refluxed for $2 \mathrm{~h}$. The completion of the reaction was monitored by TLC. The reaction mixture was poured into crushed ice to obtain a solid product. Then, the precipitate was filtered under suction, washed thoroughly with water and recrystallized from aqueous methanol. Purity and structural confirmation were done by mp and infrared (IR) spectrum. TLC solvent systems - Pet. ether:ethyl acetate (70:30).

\section{Spectral data}

5-(4-methoxyphenyl)-3-(5-methyl-3-(4-nitrophenyl)isoxazol-4yl)-4,5-dihydro-1H-pyrazole-1-carbothioamide (5a)

Yield $70 \%$, mp $121-128^{\circ} \mathrm{C}$. IR spectrum, $v, \mathrm{~cm}^{-1}: 3337,2928,1520$, $1348,1404,1095,1030 .{ }^{1} \mathrm{H}$ NMR $\left(400 \mathrm{MHz}, \mathrm{DMSO}-\mathrm{d}_{6}\right) \delta 2.60(\mathrm{~s}, 3 \mathrm{H}$, $\left.\mathrm{CH}_{3}\right), 3.0$ (dd, $1 \mathrm{H}, \mathrm{CH}_{2}$ ), 3.9 (dd, $1 \mathrm{H}, \mathrm{CH}_{2}$ ), 6.0 (td, $\left.1 \mathrm{H},-\mathrm{CH}\right), 7.00-7.31$ (m, 4H, ArH), $7.6\left(\mathrm{~s}, 1 \mathrm{H}, \mathrm{NH}_{2}\right), 7.8-8.1(\mathrm{~m}, 4 \mathrm{H}, \mathrm{ArH}), 8.2\left(\mathrm{~s}, 1 \mathrm{H}, \mathrm{NH}_{2}\right)$. 
${ }^{13} \mathrm{C}$ NMR (100 MHz, DMSO-d $) \delta 171.2,168.3,162.3,158.9,152.2$, 148.5, 138.3, 134.4, 127.5, 127.0, 125.2, 118.7, 109.7, 62.6, 56.8, 42.3, 13.1.ESI-MS: $\mathrm{m} / \mathrm{z} 438(\mathrm{M}+1)$ observed for $\mathrm{C}_{21} \mathrm{H}_{19} \mathrm{~N}_{5} \mathrm{O}_{3} \mathrm{~S}$.

5-(4-chlorophenyl)-3-(5-methyl-3-(4-nitrophenyl)isoxazol-4-yl)4,5-dihydro-1H-pyrazole-1-carbothioamide (5b)

Yield $71 \%, \mathrm{mp} 104-112^{\circ} \mathrm{C}$. IR spectrum, $v, \mathrm{~cm}^{-1}: 3349,2922,1520$, $1348,1404,1096,750 .{ }^{1} \mathrm{H}$ NMR $\left(400 \mathrm{MHz}, \mathrm{DMSO}-\mathrm{d}_{6}\right) \delta 2.6\left(\mathrm{~s}, 3 \mathrm{H}, \mathrm{CH}_{3}\right)$, $3.1\left(\mathrm{dd}, 1 \mathrm{H}, \mathrm{CH}_{2}\right), 3.9$ (dd, $\left.1 \mathrm{H}, \mathrm{CH}_{2}\right), 6.0$ (td, $\left.1 \mathrm{H},-\mathrm{CH}\right), 7.2-7.4(\mathrm{~m}, 4 \mathrm{H}, \mathrm{ArH})$, $7.6(\mathrm{~d}, 2 \mathrm{H}, \mathrm{ArH}), 7.9\left(\mathrm{~s}, 1 \mathrm{H}, \mathrm{NH}_{2}\right), 7.9-8.1(\mathrm{~m}, 4 \mathrm{H}, \mathrm{ArH}), 8.2\left(\mathrm{~s}, 1 \mathrm{H}, \mathrm{NH}_{2}\right)$.

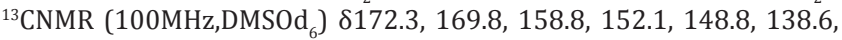
134.1, 132.8, 128.6, 127.9, 126.2, 124.8, 105.9, 62.8, 42.3, 13.3. ESIMS: m/z443 (M+1) observed for $\mathrm{C}_{20} \mathrm{H}_{16} \mathrm{ClN}_{5} \mathrm{O}_{3} \mathrm{~S}$.

3-(5-methyl-3-(4-nitrophenyl)isoxazol-4-yl)-5-phenyl-4,5dihydro-1H-pyrazole-1-carbothioamide (5c)

Yield 69\%, mp $119-125^{\circ} \mathrm{C}$. IR spectrum, $v, \mathrm{~cm}^{-1}: 3337,2924,1629,1520$, $1404,1348,1095 .{ }^{1} \mathrm{H}$ NMR $(400 \mathrm{MHz}$, DMSO-d $) \delta 2.7\left(\mathrm{~s}, 3 \mathrm{H}, \mathrm{CH}_{3}\right), 3.0$ (dd, $1 \mathrm{H}, \mathrm{CH}_{2}$ ), 3.9 (dd, $1 \mathrm{H}, \mathrm{CH}_{2}$ ), 5.9 (td, $\left.1 \mathrm{H},-\mathrm{CH}\right), 7.1-7.4(\mathrm{~m}, 5 \mathrm{H}, \mathrm{ArH})$, $7.6(\mathrm{~d}, 2 \mathrm{H}, \mathrm{ArH}), 7.7\left(\mathrm{~s}, 1 \mathrm{H}, \mathrm{NH}_{2}\right), 7.9-8.1(\mathrm{~m}, 4 \mathrm{H}, \mathrm{ArH}), 8.2\left(\mathrm{~s}, 1 \mathrm{H}, \mathrm{NH}_{2}\right)$. ${ }^{13} \mathrm{CNMR}\left(100 \mathrm{MHz}^{\mathrm{DMSOd}}{ }_{6}\right) \delta 171.9,169.2,158.8,152.2,148.6,140.9$, $134.9,128.4,126.9,126.2,124.9,123.5,109.8,62.4,42.6,13.0 . E S I-M S$ : $\mathrm{m} / \mathrm{z} 408(\mathrm{M}+1)$ observed for $\mathrm{C}_{20} \mathrm{H}_{17} \mathrm{~N}_{5} \mathrm{O}_{3} \mathrm{~S}$.

5-(4-bromophenyl)-3-(5-methyl-3-(4-nitrophenyl)isoxazol-4-yl)4,5-dihydro-1H-pyrazole-1-carbothioamide (5d)

yield $70 \%, \mathrm{mp} 117-124^{\circ} \mathrm{C}$. IR spectrum, $v, \mathrm{~cm}^{-1}: 3349,2929,1520$, 1348, 1404, 1240, 1095, 668. ${ }^{1} \mathrm{H}$ NMR (400 MHz, DMSO-d 6 ) $\delta 2.8$ (s, $\left.3 \mathrm{H}, \mathrm{CH}_{3}\right), 3.1\left(\mathrm{dd}, 1 \mathrm{H}, \mathrm{CH}_{2}\right), 4.1\left(\mathrm{dd}, 1 \mathrm{H}, \mathrm{CH}_{2}\right), 5.9(\mathrm{td}, 1 \mathrm{H}, \mathrm{CH}), 7.1-$ $7.5(\mathrm{~m}, 4 \mathrm{H}, \mathrm{ArH}), 7.7\left(\mathrm{~s}, 1 \mathrm{H}, \mathrm{NH}_{2}\right), 7.8-8.1(\mathrm{~m}, 4 \mathrm{H}, \mathrm{ArH}), 8.2\left(\mathrm{~s}, 1 \mathrm{H}, \mathrm{NH}_{2}\right)$. ${ }^{13}{ }^{13}$ NMR $\left(100 \mathrm{MHz}\right.$, DMSOd $\left._{6}\right) \delta 171.9,169.4,158.9,152.5,148.2,141.2$, $136.4,130.2,127.8,126.8,126.4,124.7,105.8,62.6,42.1,13.4$. ESI-MS: $\mathrm{m} / \mathrm{z} 486(\mathrm{M}+1)$ observed for $\mathrm{C}_{20} \mathrm{H}_{16} \mathrm{BrN}_{5} \mathrm{O}_{3} \mathrm{~S}$.

3-(5-methyl-3-(4-nitrophenyl)isoxazol-4-yl)-5-(p-tolyl)-4,5dihydro-1H-pyrazole-1-carbothioamide (5e)

Yield $72 \%$, mp $125-137^{\circ} \mathrm{C}$. IR spectrum, $v, \mathrm{~cm}^{-1}: 3346,2923,1520$, $14041348,1095 .{ }^{1} \mathrm{H}$ NMR $\left(400 \mathrm{MHz}, \mathrm{DMSO}-\mathrm{d}_{6}\right) \delta 2.6\left(\mathrm{~s}, 3 \mathrm{H}, \mathrm{CH}_{3}\right), 2.8$ (s, 3H, $\mathrm{CH}_{3}$ ), $3.2\left(\mathrm{dd}, 1 \mathrm{H}, \mathrm{CH}_{2}\right.$ ), $4.0\left(\mathrm{dd}, 1 \mathrm{H}, \mathrm{CH}_{2}\right), 6.0(\mathrm{td}, 1 \mathrm{H},-\mathrm{CH}), 7.1-7.4$ (m, 4H, ArH), $7.6\left(\mathrm{~s}, 1 \mathrm{H}, \mathrm{NH}_{2}\right), 7.9-8.1(\mathrm{~m}, 4 \mathrm{H}, \mathrm{ArH}), 8.2\left(\mathrm{~s}, 1 \mathrm{H}, \mathrm{NH}_{2}\right)$. ${ }^{13} \mathrm{C}$ NMR $\left(100 \mathrm{MHz}\right.$, DMSO-d $\left.\mathrm{d}_{6}\right) \delta 171.8,169.4,158.4,152.8,148.2,138.1$, 136.6, 134.8, 128.5, 126.4, 125.1, 123.3, 109.5, 62.4, 42.4, 21.5, 13.1 . ESI-MS: $\mathrm{m} / \mathrm{z} 422(\mathrm{M}+1)$ observed for $\mathrm{C}_{21} \mathrm{H}_{19} \mathrm{~N}_{5} \mathrm{O}_{3} \mathrm{~S}$.

5-(4-fluorophenyl)-3-(5-methyl-3-(4-nitrophenyl) isoxazol-4-yl)4,5-dihydro-1H-pyrazole-1-carbothioamide (5f)

Yield $70 \%, \mathrm{mp} 110-116^{\circ} \mathrm{C}$. IR spectrum, v, $\mathrm{cm}^{-1}: 3349,2993,1520$, $1404,1348,1095,1000 .{ }^{1} \mathrm{H}$ NMR (400 MHz, DMSO-d 6 ) $\delta 2.7\left(\mathrm{~s}, 3 \mathrm{H}^{-\mathrm{CH}_{3}}\right.$ ), 3.0 (dd, $1 \mathrm{H}, \mathrm{CH}_{2}$ ), 3.9 (dd, $1 \mathrm{H}, \mathrm{CH}_{2}$ ), 5.9 (td, $\left.1 \mathrm{H},-\mathrm{CH}\right), 7.2-7.5(\mathrm{~m}, 4 \mathrm{H}$, $\mathrm{ArH}), 7.7\left(\mathrm{~s}, 1 \mathrm{H}, \mathrm{NH}_{2}\right), 7.8-8.0(\mathrm{~m}, 4 \mathrm{H}, \mathrm{ArH}), 8.2\left(\mathrm{~s}, 1 \mathrm{H}, \mathrm{NH}_{2}\right)$. ESI-MS: $\mathrm{m} /$ z426 $(\mathrm{M}+1)$ observed for $\mathrm{C}_{20} \mathrm{H}_{16} \mathrm{FN}_{5} \mathrm{O}_{3} \mathrm{~S} \cdot{ }^{13} \mathrm{C}$ NMR (100 MHz, DMSO-d $\left.\mathrm{d}_{6}\right)$ $\delta 172.2,169.5,161.1,158.8,152.3,148.5,138.7,134.6,128.9,126.7$, 125.9, 118.3, 109.2, 62.8, 42.3, 13.1. ESI-MS: m/z $471(\mathrm{M}+1)$ observed for $\mathrm{C}_{26} \mathrm{H}_{19} \mathrm{FN}_{4} \mathrm{O}_{4}$.

5-(2-chlorophenyl)-N-methyl-3-(5-methyl-3-(4-nitrophenyl) isoxazol-4-yl)-4,5-dihydro-1H-pyrazole-1-carbothioamide (5g)

Yield 69\%, mp 96-108 ${ }^{\circ} \mathrm{C}$. IR spectrum, $v, \mathrm{~cm}^{-1}: 3346,2925,1520,1404$, 1348, 1095, 750. ${ }^{1} \mathrm{H}$ NMR (400 MHz, DMSO-d $) \delta 2.7$ (s, 3H, $\mathrm{CH}_{3}$ ), 3.0 (dd, $1 \mathrm{H}, \mathrm{CH}_{2}$ ), 4.0 (dd, $1 \mathrm{H}, \mathrm{CH}_{2}$ ), 5.9 (td, $\left.1 \mathrm{H},-\mathrm{CH}\right), 7.2-7.5(\mathrm{~m}, 4 \mathrm{H}, \mathrm{ArH})$, 7.7 (s, $\left.1 \mathrm{H}, \mathrm{NH}_{2}\right), 7.8-8.1(\mathrm{~m}, 4 \mathrm{H}, \mathrm{ArH}), 8.2\left(\mathrm{~s}, 1 \mathrm{H}, \mathrm{NH}_{2}\right) .{ }^{13} \mathrm{C}$ NMR $(100$ MHz, DMSO-d ${ }_{6}$ ) $\delta 172.5,169.6,158.9,152.7,148.6,139.3,134.2,132.5$, 128.6, 128.2, 126.7, 124.9, 109.2, 62.8, 45.1, 13.2. ESI-MS: m/z443 $(\mathrm{M}+1)$ observed for $\mathrm{C}_{20} \mathrm{H}_{16} \mathrm{ClN}_{5} \mathrm{O}_{3} \mathrm{~S}$. 3-(5-methyl-3-(4-nitrophenyl)isoxazol-4-yl)-5-(thiophen-2-yl)4,5-dihydro-1H-pyrazole-1-carbothioamide (5h)

Yield $74 \%, \mathrm{mp} 104-112^{\circ} \mathrm{C}$. IR spectrum, $v, \mathrm{~cm}^{-1}: 3348,2993,1520$, $1404,1348,1095 .{ }^{1} \mathrm{H}$ NMR $\left(400 \mathrm{MHz}, \mathrm{DMSO}-\mathrm{d}_{6}\right) \delta 2.7\left(\mathrm{~s}, 3 \mathrm{H}, \mathrm{CH}_{3}\right)$, $3.1\left(\mathrm{dd}, 1 \mathrm{H}, \mathrm{CH}_{2}\right), 4.0\left(\mathrm{dd}, 1 \mathrm{H}, \mathrm{CH}_{2}\right), 6.0(\mathrm{td}, 1 \mathrm{H},-\mathrm{CH}), 6.9-7.4(\mathrm{~m}$, $3 \mathrm{H}, \mathrm{ArH}), 7.7\left(\mathrm{~s}, 1 \mathrm{H}, \mathrm{NH}_{2}\right), 7.8-8.1(\mathrm{~m}, 4 \mathrm{H}, \mathrm{ArH}), 8.2\left(\mathrm{~s}, 1 \mathrm{H}, \mathrm{NH}_{2}\right) .{ }^{13} \mathrm{C}$ NMR (100 MHz, DMSO-d ${ }_{6}$ ) $\delta 171.8,169.8,158.9,152.4,148.5,138.3$, 135.5, 128.4, 127.5, 126.5, 126.0, 123.6, 121.5, 109.3, 62.3, 45.0, 13.1. ESI-MS: $\mathrm{m} / \mathrm{z} 414(\mathrm{M}+1)$ observed for $\mathrm{C}_{18} \mathrm{H}_{15} \mathrm{~N}_{5} \mathrm{O}_{3} \mathrm{~S}_{2}$.

5-(furan-2-yl)-3-(5-methyl-3-(4-nitrophenyl)isoxazol-4-yl)-4,5dihydro-1H-pyrazole-1-carbothioamide(5i)

Yield $71 \%, \mathrm{mp} 105-118^{\circ} \mathrm{C}$. IR spectrum, $v, \mathrm{~cm}^{-1}: 3346,2925,1520$, $1404,1348,1095 .{ }^{1} \mathrm{H}$ NMR (400 MHz, DMSO-d $) \delta 2.60\left(\mathrm{~s}, 3 \mathrm{H}, \mathrm{CH}_{3}\right.$ ), 3.0 (dd, $\left.1 \mathrm{H}, \mathrm{CH}_{2}\right), 4.0$ (dd, $1 \mathrm{H}, \mathrm{CH}_{2}$ ), 6.0 (td, $\left.1 \mathrm{H},-\mathrm{CH}\right), 6.8-7.4(\mathrm{~m}, 3 \mathrm{H}, \mathrm{ArH})$, $7.6\left(\mathrm{~s}, 1 \mathrm{H}, \mathrm{NH}_{2}\right), 7.9-8.1(\mathrm{~m}, 4 \mathrm{H}, \mathrm{ArH}), 8.2\left(\mathrm{~s}, 1 \mathrm{H}, \mathrm{NH}_{2}\right) .{ }^{13} \mathrm{C}$ NMR $(100$ MHz, DMSO-d $)$ ) $\delta 171.8,169.2,158.9,152.7,150.8,148.5,142.4,135.5$, 126.5, 123.6, 112.6, 113.9, 109.3, 60.1, 42.1, 13.1. ESI-MS: m/z398 $(\mathrm{M}+1)$ observed for $\mathrm{C}_{18} \mathrm{H}_{15} \mathrm{~N}_{5} \mathrm{O}_{4} \mathrm{~S}$.

5-(4-methoxyphenyl) -N-methyl-3-(5-methyl-3-(4-nitrophenyl) isoxazol-4-yl) -4,5-dihydro-1H-pyrazole-1-carbothioamide (5j)

Yield $68 \%$, mp $124-132^{\circ} \mathrm{C}$. IR spectrum, $v, \mathrm{~cm}^{-1}: 3337,2927,1525$, $1348,1404,1095,1032 .{ }^{1} \mathrm{H}$ NMR $\left(400 \mathrm{MHz}, \mathrm{DMSO}-\mathrm{d}_{6}\right) \delta 2.6\left(\mathrm{~s}, 3 \mathrm{H}, \mathrm{CH}_{3}\right)$, $2.8\left(\mathrm{~s}, 3 \mathrm{H}, \mathrm{CH}_{3}\right), 3.0\left(\mathrm{dd}, 1 \mathrm{H}, \mathrm{CH}_{2}\right), 3.6\left(\mathrm{~s}, 3 \mathrm{H}, \mathrm{OCH}_{3}\right) 4.0\left(\mathrm{dd}, 1 \mathrm{H}, \mathrm{CH}_{2}\right), 6.0$ (td, 1H, -CH), 7.1-7.4 (m, 4H, ArH), $7.7\left(\mathrm{~s}, 1 \mathrm{H}, \mathrm{NH}_{2}\right), 7.9-8.1(\mathrm{~m}, 4 \mathrm{H}, \mathrm{ArH})$, $8.2\left(\mathrm{~s}, 1 \mathrm{H}, \mathrm{NH}_{2}\right) \cdot{ }^{13} \mathrm{C}$ NMR $(100 \mathrm{MHz}$, DMSO-d 6 ) $\delta 172.0,169.1,162.5$, $158.3,152.2,148.7,138.3,134.4,127.8,127.3,124.8,118.4,109.4,62.2$, 56.8, 42.7, 31.1 13.1. ESI-MS: $\mathrm{m} / \mathrm{z} 451(\mathrm{M}+1)$ observed for $\mathrm{C}_{22} \mathrm{H}_{21} \mathrm{~N}_{5} \mathrm{O}_{4} \mathrm{~S}$.

5-(4-chlorophenyl)-N-methyl-3-(5-methyl-3-(4-nitrophenyl) isoxazol-4-yl)-4,5-dihydro-1H-pyrazole-1-carbothioamide (5k) Yield $70 \%, \mathrm{mp} 121-123^{\circ} \mathrm{C}$. IR spectrum, $v, \mathrm{~cm}^{-1}: 3339,2925,1520$, $1404,1348,1095,750 .{ }^{1} \mathrm{H}$ NMR $\left(400 \mathrm{MHz}\right.$, DMSO-d $\left.\mathrm{d}_{6}\right) \delta 2.6\left(\mathrm{~s}, 3 \mathrm{H}_{1} \mathrm{CH}_{3}\right)$, $2.8\left(\mathrm{~s}, 3 \mathrm{H}, \mathrm{CH}_{3}\right), 3.0\left(\mathrm{dd}, 1 \mathrm{H}, \mathrm{CH}_{2}\right), 4.0\left(\mathrm{dd}, 1 \mathrm{H}, \mathrm{CH}_{2}\right), 5.9(\mathrm{td}, 1 \mathrm{H},-\mathrm{CH}), 7.2-$ $7.5(\mathrm{~m}, 4 \mathrm{H}, \mathrm{ArH}), 7.7\left(\mathrm{~s}, 1 \mathrm{H}, \mathrm{NH}_{2}\right), 7.8-8.1(\mathrm{~m}, 4 \mathrm{H}, \mathrm{ArH}), 8.2\left(\mathrm{~s}, 1 \mathrm{H}, \mathrm{NH}_{2}\right)$.

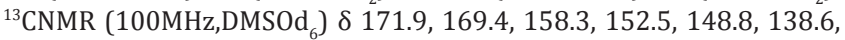
134.5, 132.2, 128.3, 127.5, 126.7, 124.4, 105.7, 62.7, 45.1, 31.4, 13.3. ESI-MS: m/z457 (M+1) observed for $\mathrm{C}_{21} \mathrm{H}_{18} \mathrm{ClN}_{5} \mathrm{O}_{3} \mathrm{~S}$.

$N$-methyl-3-(5-methyl-3-(4-nitrophenyl)isoxazol-4-yl)-5-phenyl4,5-dihydro-1H-pyrazole-1-carbothioamide (5l)

Yield $71 \%$, mp $117-123^{\circ} \mathrm{C}$. IR spectrum, v, $\mathrm{cm}^{-1}: 3342,2925,1521$, 1404, 1346,1095. ${ }^{1} \mathrm{H}$ NMR $(400 \mathrm{MHz}$, DMSO-d $) \delta 2.5\left(\mathrm{~s}, 3 \mathrm{H}_{6} \mathrm{CH}_{3}\right)$, 2.8(s, $\left.3 \mathrm{H}, \mathrm{CH}_{3}\right), 3.1\left(\mathrm{dd}, 1 \mathrm{H}, \mathrm{CH}_{2}\right), 4.0\left(\mathrm{dd}, 1 \mathrm{H}, \mathrm{CH}_{2}\right), 5.9(\mathrm{td}, 1 \mathrm{H},-\mathrm{CH}), 7.1-$ $7.4(\mathrm{~m}, 4 \mathrm{H}, \mathrm{ArH}), 7.7\left(\mathrm{~s}, 1 \mathrm{H}, \mathrm{NH}_{2}\right), 7.8-8.1(\mathrm{~m}, 4 \mathrm{H}, \mathrm{ArH}), 8.2\left(\mathrm{~s}, 1 \mathrm{H}, \mathrm{NH}_{2}\right)$ .${ }^{13}$ CNMR (100MHz,DMSOd $)_{6} \delta 172.4,168.9,158.4,152.7,148.9,141.1$, 136.2, 128.9, 126.8, 126.2, 124.6, 123.5, 109.2, 62.1, 45.6, 13.0, 31.6. ESI-MS: $\mathrm{m} / \mathrm{z} 422(\mathrm{M}+1)$ observed for $\mathrm{C}_{21} \mathrm{H}_{19} \mathrm{~N}_{5} \mathrm{O}_{3} \mathrm{~S}$.

5-(4-bromophenyl)-N-methyl-3-(5-methyl-3-(4-nitrophenyl) isoxazol-4-yl)-4,5-dihydro-1H-pyrazole-1-carbothioamide (5m) Yield $69 \%, \mathrm{mp} 111-117^{\circ} \mathrm{C}$. IR spectrum, $v, \mathrm{~cm}^{-1}: 3340,2926,1523$, $1404,1348,1093,670 .{ }^{1} \mathrm{H}$ NMR $(400 \mathrm{MHz}$, DMSO-d $) \delta 2.6\left(\mathrm{~s}, 3 \mathrm{H}_{6} \mathrm{CH}_{3}\right)$, $2.8\left(\mathrm{~s}, 3 \mathrm{H}, \mathrm{CH}_{3}\right), 3.1\left(\mathrm{dd}, 1 \mathrm{H}, \mathrm{CH}_{2}\right), 3.0\left(\mathrm{dd}, 1 \mathrm{H}, \mathrm{CH}_{2}\right), 5.9(\mathrm{td}, 1 \mathrm{H},-\mathrm{CH}), 7.2-$ $7.5(\mathrm{~m}, 4 \mathrm{H}, \mathrm{ArH}), 7.7\left(\mathrm{~s}, 1 \mathrm{H}, \mathrm{NH}_{2}\right), 7.8-8.1(\mathrm{~m}, 4 \mathrm{H}, \mathrm{ArH}), 8.2\left(\mathrm{~s}, 1 \mathrm{H}, \mathrm{NH}_{2}\right)$. ${ }^{13} \mathrm{CNMR}\left(100 \mathrm{MHz}, \mathrm{DMSOd}_{6}\right) \delta 172.5,168.9,158.6,152.3,148.8,140.9$, 136.8, 130.9, 128.4, 126.4, 124.7, 122.3, 105.9, 62.4, 42.9, 31.6, 13.4 . ESI-MS: m/z500 (M+1) observed for $\mathrm{C}_{21} \mathrm{H}_{18} \mathrm{BrN}_{5} \mathrm{O}_{3} \mathrm{~S}$

5-(4-fluorophenyl)-N-methyl-3-(5-methyl-3-(4-nitrophenyl) isoxazol-4-yl)-4,5-dihydro-1H-pyrazole-1-carbothioamide (5n)

Yield $72 \%, \mathrm{mp} 115-120^{\circ} \mathrm{C}$. IR spectrum, $v, \mathrm{~cm}^{-1}: 3344,2923,1525$, $1404,1348,1096,1000 .{ }^{1} \mathrm{H}$ NMR $(400 \mathrm{MHz}$, DMSO-d 6$) \delta 2.7\left(\mathrm{~s}, 3 \mathrm{H}_{6} \mathrm{CH}_{3}\right)$, 


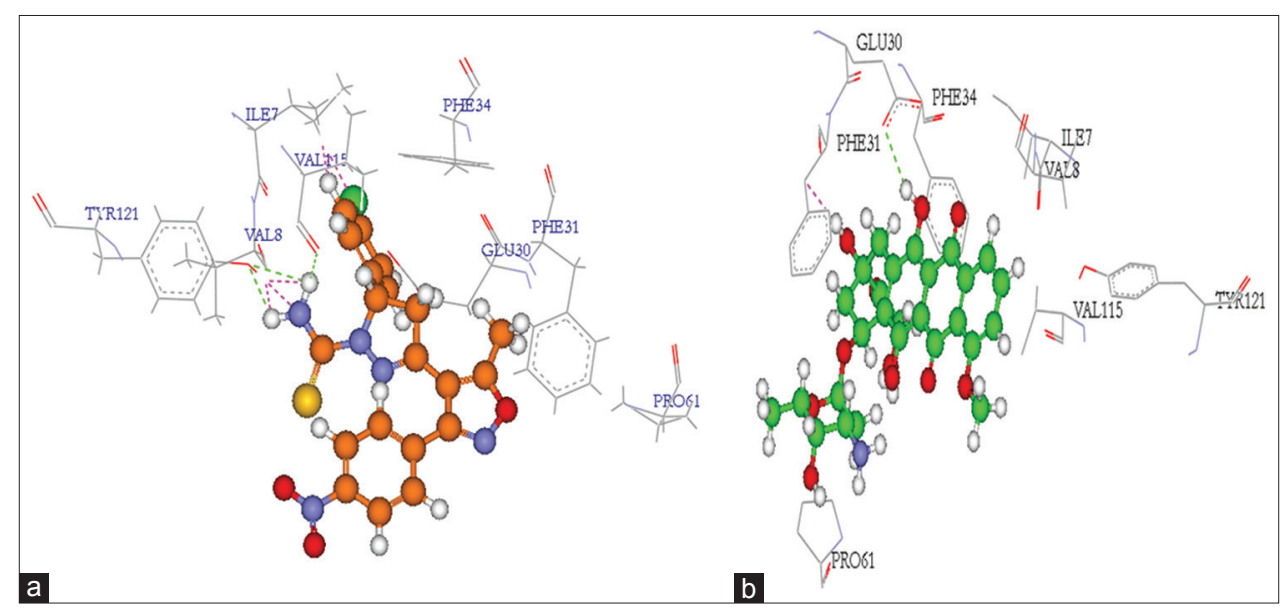

Fig. 2: The docking conformation of (a) compounds $5 \mathrm{~b}$ and (b) the reference drug doxorubicin inside the protein human DHFR (1KMS) binding site

$2.8\left(\mathrm{~s}, 3 \mathrm{H}, \mathrm{CH}_{3}\right), 3.0\left(\mathrm{dd}, 1 \mathrm{H}, \mathrm{CH}_{2}\right), 4.1\left(\mathrm{dd}, 1 \mathrm{H}, \mathrm{CH}_{2}\right), 6.0(\mathrm{td}, 1 \mathrm{H},-\mathrm{CH}), 7.1-$ $7.5(\mathrm{~m}, 4 \mathrm{H}, \mathrm{ArH}), 7.8\left(\mathrm{~s}, 1 \mathrm{H}, \mathrm{NH}_{2}\right), 7.9-8.1(\mathrm{~m}, 4 \mathrm{H}, \mathrm{ArH}), 8.2\left(\mathrm{~s}, 1 \mathrm{H}, \mathrm{NH}_{2}\right)$. ${ }^{13} \mathrm{C}$ NMR $(100 \mathrm{MHz}$, DMSO-d $) \delta 171.6,168.8,161.4,158.3,152.8,148.9$, 137.4, 134.6, 128.2, 126.7, 125.5, 118.6,109.6, 62.1, 45.0, 31.6,13.1.ESIMS: $\mathrm{m} / \mathrm{z} 440(\mathrm{M}+1)$ observed for $\mathrm{C}_{21} \mathrm{H}_{18} \mathrm{FN}_{5} \mathrm{O}_{3} \mathrm{~S}$.

N-methyl-3-(5-methyl-3-(4-nitrophenyl)isoxazol-4-yl)-5-(ptolyl)-4,5-dihydro-1H-pyrazole-1-carbothioamide (5o)

Yield $69 \%$, mp $119-125^{\circ} \mathrm{C}$. IR spectrum, v, $\mathrm{cm}^{-1}: 3340,2926,1523$, $1404,1348,1093$.

${ }^{1} \mathrm{H}$ NMR (400 MHz, DMSO-d $) \delta 2.6\left(\mathrm{~s}, 3 \mathrm{H}, \mathrm{CH}_{3}\right), 2.8\left(\mathrm{~s}, 3 \mathrm{H}, \mathrm{CH}_{3}\right), 3.1(\mathrm{dd}$, $\left.1 \mathrm{H}, \mathrm{CH}_{2}\right), 4.1\left(\mathrm{dd}, 1 \mathrm{H}, \mathrm{CH}_{2}\right), 5.9(\mathrm{td}, 1 \mathrm{H}, \mathrm{CH}), 7.1-7.5(\mathrm{~m}, 4 \mathrm{H}, \mathrm{ArH}), 7.7(\mathrm{~s}$, $\left.1 \mathrm{H}, \mathrm{NH}_{2}\right), 7.8-8.1(\mathrm{~m}, 4 \mathrm{H}, \mathrm{ArH}), 8.2\left(\mathrm{~s}, 1 \mathrm{H}, \mathrm{NH}_{2}\right) \cdot{ }^{13} \mathrm{C}$ NMR $(100 \mathrm{MHz}$, DMSO-d ${ }_{6}$ ) $\delta 172.3,169.5,158.2,152.1,148.6,138.5,136.1,134.4,128.2$, 126.5,125.6, 123.3, 109.8, 61.9, 42.8, 31.9, 21.5, 13.1. ESI-MS: m/z436 $(\mathrm{M}+1)$ observed for $\mathrm{C}_{22} \mathrm{H}_{21} \mathrm{~N}_{5} \mathrm{O}_{3} \mathrm{~S}$.

5-(2-chlorophenyl) -N-methyl-3-(5-methyl-3-(4-nitrophenyl) isoxazol-4-yl) -4,5-dihydro-1H-pyrazole-1-carbothioamide (5p) Yield $73 \%$, mp $121-123^{\circ} \mathrm{C}$. IR spectrum, $v, \mathrm{~cm}^{-1}: 3342,2928,1523$, $1404,1348,1095,752$.

${ }^{1} \mathrm{H}$ NMR (400 MHz, DMSO-d $) \delta 2.5\left(\mathrm{~s}, 3 \mathrm{H}, \mathrm{CH}_{3}\right), 2.7$ (s, 3H, $\mathrm{CH}_{3}$ ), 3.0(dd, $\left.1 \mathrm{H}, \mathrm{CH}_{2}\right), 4.1\left(\mathrm{dd}, 1 \mathrm{H}, \mathrm{CH}_{2}\right), 5.9(\mathrm{td}, 1 \mathrm{H}, \mathrm{CH}), 7.1-7.5(\mathrm{~m}, 4 \mathrm{H}, \mathrm{ArH}), 7.6(\mathrm{~s}, 1 \mathrm{H}$, $\mathrm{NH}_{2}$ ), 7.9-8.1 (m, 4H, ArH), 8.2(s,1H, $\mathrm{NH}_{2}$ ). $\delta 171.8,168.6,158.2,152.2$, 148.8, 138.8, 135.2, 131.9, 128.9, 128.1, 126.5, 124.1, 109.8, 62.2, 45.6, 21.7, 31.2, 13.2. ESI-MS: $\mathrm{m} / \mathrm{z} 457(\mathrm{M}+1)$ observed for $\mathrm{C}_{22} \mathrm{H}_{21} \mathrm{ClN}_{5} \mathrm{O}_{3} \mathrm{~S}$.

N-methyl-3-(5-methyl-3-(4-nitrophenyl)isoxazol-4-yl)-5(thiophen-2-yl)-4,5-dihydro-1H-pyrazole-1-carbothioamide(5q)

Yield $72 \%, \mathrm{mp} 105-114^{\circ} \mathrm{C}$. IR spectrum, $v, \mathrm{~cm}^{-1}: 3339,2927,1526$, $1404,1348,1097$.

${ }^{1} \mathrm{H}$ NMR ( $400 \mathrm{MHz}$, DMSO-d $) \delta 2.6\left(\mathrm{~s}, 3 \mathrm{H}, \mathrm{CH}_{3}\right), 2.8\left(\mathrm{~s}, 3 \mathrm{H}, \mathrm{CH}_{3}\right), 3.1(\mathrm{dd}, 1 \mathrm{H}$, $\left.\mathrm{CH}_{2}\right), 3.9\left(\mathrm{dd}, 1 \mathrm{H}, \mathrm{CH}_{2}\right), 6.0(\mathrm{td}, 1 \mathrm{H}, \mathrm{CH}), 6.9-7.4(\mathrm{~m}, 3 \mathrm{H}, \mathrm{ArH}), 7.8\left(\mathrm{~s}, 1 \mathrm{H}, \mathrm{NH}_{2}\right)$, 7.9-8.1 (m, 4H, ArH), 8.2(s,1H,NH $)_{2}$. ESI-MS: m/z428 (M+1) observed for $\mathrm{C}_{19} \mathrm{H}_{17} \mathrm{~N}_{5} \mathrm{O}_{3} \mathrm{~S}_{2} .8171 .8,169.8,158.9,153.9,148.5,138.7,134.9,128.8,127.9$, $126.8,126.1,123.4,121.4,109.7,62.6,45.4,13.0$.

5-(furan-2-yl)-N-methyl-3-(5-methyl-3-(4-nitrophenyl)isoxazol4-yl)-4,5-dihydro-1H-pyrazole-1-carbothioamide(5r)

Yield $70 \%$, mp $117-123^{\circ} \mathrm{C}$. IR spectrum, $v, \mathrm{~cm}^{-1}: 3342,2925,1521$, $1404,1346,1095 .{ }^{1} \mathrm{H}$ NMR (400 MHz, DMSO-d $) \delta 2.5\left(\mathrm{~s}, 3 \mathrm{H}, \mathrm{CH}_{3}\right.$ ),

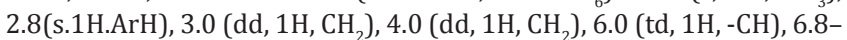
$7.4(\mathrm{~m}, 3 \mathrm{H}, \mathrm{ArH}), 7.7\left(\mathrm{~s}, 1 \mathrm{H}, \mathrm{NH}_{2}\right), 7.9-8.1(\mathrm{~m}, 4 \mathrm{H}, \mathrm{ArH}), 8.2\left(\mathrm{~s}, 1 \mathrm{H}, \mathrm{NH}_{2}\right)$.
${ }^{13}$ CNMR(100MHz,DMSO-d $) \delta 172.4,169.5,158.9,153.5,150.2,148.8$, $142.6,135.8,126.3,123.5,112.4,113.7,109.6,62.6,42.6,31.6,13.1$.ESIMS: $\mathrm{m} / \mathrm{z} 411(\mathrm{M}+1)$ observed for $\mathrm{C}_{19} \mathrm{H}_{17} \mathrm{~N}_{5} \mathrm{O}_{4} \mathrm{~S}$.

\section{Docking studies}

The molecular docking application is used to interpret the binding affinities and interaction modes of synthesized compounds and the target protein DHFR implementing the LibDock module of DS. The LibDock docking algorithm is a site-featured procedure. The binding site features are referred to as "Hot Spots" and that are determined in a grid settled inside the active site. This enables the hotspot map count in the active site of the protein for a polar and polar cluster that enables considerable alignment of the ligand arrangement to protein interaction sites. The minimized protein and ligand along with the binding site atom number or the X, Y, and Z points of the binding site residue within $12 \AA$ submitted to the LibDock setup. All other docking and resultant scoring parameters applied were executed at their default settings. Finally, it restores the entire minimized ligand poses and their rankings based on the scoring function that calculates the binding affinity score or the docking score (LibDock score) of the protein-ligand complex. Furthermore, the possible binding energies, hydrogen bonding, and various interaction poses are calculated. The binding poses were recommended on the criterion of LibDock Score rank. Binding poses with the highest LibDock score and lowest binding energy are preferred as the best pose and further binding interactions of the best pose for each compound are analyzed. In addition, the Analyze Ligand Poses subprotocol in DS was applied. To ensure the docking method was efficient, docking the standard reference drug doxorubicin with the human DHFR binding site is done. The docking results are examined in comparison to that of the reference doxorubicin in terms of their interactions and docking scores with the protein DHFR.

\section{RESULTS AND DISCUSSION}

\section{Chemistry}

A series of 5-(substituted phenyl) -3-(5-methyl-3-(4-nitrophenyl) isoxazole-4-yl) -4,5-dihydro-1H-pyrazole-1-carbothioamide and 5-(substituted phenyl) -N-methyl-3-(5-methyl-3-(4-nitrophenyl) isoxazole-4-yl) -4,5-dihydro-1H-pyrazole-1-carbothioamide $\mathbf{5}$ (a-r) were synthesized in following five steps (Fig. 1). In the first step, 4-nitrobenzaldehydeoxime 1 was prepared by the reaction of 4-nitrobenzaldehyde with hydroxylamine hydrochloride. In the second step, 1-(5-methyl-3-(4-nitrophenyl) isoxazole-4-yl) ethanone3 was prepared from in situ synthesized N-hydroxy-4-nitrobenzimidoyl chloride $\mathbf{2}$ and acetylacetone in methanol. In the next step, the compound 3 was condensed with various substituted aromatic aldehydes in the presence of $\mathrm{NaOH}$ under reflux for 30-50 min to obtain intermediate 1-(5-methyl-3-(4-nitrophenyl) isoxazole-4-yl) -3-(substituted phenyl) prop-2-en-1-one $\mathbf{4}$ (a-m). In the final step, intermediate $\mathbf{4}(\mathbf{a}-\mathbf{m})$ was refluxed with thiosemicarbazides and substituted thiosemicarbazide 
Table 1: Calculated docking scores, interacting atoms and amino acids and H-bond count of synthesized compounds along with reference drug doxorubicin with the human DHFR (1KMS) active site

\begin{tabular}{|c|c|c|c|}
\hline Name & LibDock score & Interacting atoms & H-bond count \\
\hline \multirow[t]{2}{*}{$5 a$} & 125.036 & H45 - A: VAL115:0 & 3 \\
\hline & & H42 - A: VAL8: HG22 & \\
\hline \multirow[t]{6}{*}{$5 b$} & 144.368 & H45 - A: VAL115:0 & 4 \\
\hline & & H45 - A: TYR121: OH & \\
\hline & & H46 - A: TYR121: OH & \\
\hline & & N25 - A: TYR121: HH & \\
\hline & & H46 - A: TYR121: HH & \\
\hline & & N25 - A: TYR121: HH & \\
\hline \multirow[t]{2}{*}{$5 c$} & 125.036 & H48 - A: PRO61: CG & 3 \\
\hline & & C22 - A: PRO61: HG1 & \\
\hline \multirow[t]{2}{*}{$5 d$} & 126.851 & H39 - A: PRO61: HD2 & 1 \\
\hline & & H37 - A: PRO61: HD1 & \\
\hline \multirow[t]{6}{*}{$5 e$} & 134.368 & H41 - A: PHE31: HZ & 4 \\
\hline & & H39 - A: PRO61: HD2 & \\
\hline & & H37 - A: PRO61: HD1 & \\
\hline & & N25 - A: TYR121: HH & \\
\hline & & H46 - A: TYR121: HH & \\
\hline & & N25 - A: TYR121: HH & \\
\hline \multirow[t]{4}{*}{$5 \mathrm{f}$} & 134.65 & F: H41 - A: VAL115:0 & 3 \\
\hline & & H42 - A: TYR121: OH & \\
\hline & & N19 - A: TYR121: HH & \\
\hline & & H42 - A: TYR121: HH & \\
\hline \multirow[t]{2}{*}{$5 \mathrm{~g}$} & 132.17 & H48 - A: PRO61: CG & 1 \\
\hline & & C22 - A: PRO61: HG1 & \\
\hline \multirow[t]{4}{*}{$5 \mathrm{~h}$} & 142.302 & H41 - A: VAL115:0 & 3 \\
\hline & & H42 - A: TYR121: OH & \\
\hline & & N19 - A: TYR121: HH & \\
\hline & & H42 - A: TYR121: HH & \\
\hline \multirow[t]{2}{*}{$5 \mathrm{i}$} & 134.65 & A: TYR121: HH: N8 & 2 \\
\hline & & 09 - A: VAL8: HA & \\
\hline $5 \mathrm{j}$ & 128.119 & A: TYR121: HH: 09 & 2 \\
\hline \multirow[t]{4}{*}{$5 \mathrm{k}$} & 122.242 & H39 - A: VAL115:0 & 3 \\
\hline & & A: TYR121: HH - 025 & \\
\hline & & A: VAL8: HG22 - C24 & \\
\hline & & A: VAL8: HG22 - H43 & \\
\hline 51 & 124.452 & A: TYR121: HH: 09 & 2 \\
\hline $5 \mathrm{~m}$ & 127.339 & A: TYR121: HH: 09 & 2 \\
\hline $5 n$ & 132.451 & A: TYR121: HH: 09 & 2 \\
\hline \multirow[t]{2}{*}{50} & 132.17 & A: PR061: HD1 -: H38 & 1 \\
\hline & & A: PR061: HD2 -: H40 & \\
\hline \multirow[t]{2}{*}{$5 p$} & 112.318 & H48 - A: PR061: CG & 1 \\
\hline & & C22 - A: PR061: HG1 & \\
\hline \multirow[t]{3}{*}{$5 q$} & 123.995 & H39 - A: VAL115:0 & 2 \\
\hline & & H42 - A: VAL8: HA & \\
\hline & & C24 - A: VAL8: HG22 & \\
\hline \multirow[t]{2}{*}{$5 r$} & 128.119 & A: TYR121: HH: N8 & 2 \\
\hline & & 09 - A: VAL8: HA & \\
\hline Doxorubicin & 150.181 & Glu30, phe34, phe31, ile7, val115, tyr121, val8, pro61 & 1 \\
\hline
\end{tabular}

in potassium hydroxide using ethanol as a solvent for 3-4 h to result in 5-(substituted phenyl) -3-(5-methyl-3-(4-nitrophenyl) isoxazole4-yl) -4,5-dihydro-1H-pyrazole-1-carbothioamide and 5-(substituted phenyl) -N-methyl-3-(5-methyl-3-(4-nitrophenyl) isoxazole-4-yl) -4,5-dihydro-1H-pyrazole-1-carbothioamide 5 (a-r) were synthesized in quantitative yields in quantitative yields. All the derivatives were characterized by ${ }^{1} \mathrm{H}$ NMR, IR, and ESI-MS spectra.

\section{Docking studies}

Molecular docking was performed using DHFR protein and synthesized compounds 5 (a-r) in LibDock. Different poses were generated for each ligand and scored using a LibDock scoring function which estimates their equitable LibDock scores with various orientations. Based on the docked score, all the compounds were ranked. The docked complex of protein and compounds was forming hydrogen bonds and other parameters like Van der Waals clashes. Finally, Analyze Ligand Poses subprotocol was executed to list out $\mathrm{H}$ bonds and close contacts (Van der Waals clashes) between docked complexes. The interacting residues were analyzed using the receptor-ligand interaction protocol of DS. Table 1 shows the calculated binding scores of the compounds inward the human DHFR active site. It was observed that out of all compounds, the compound $5 \mathrm{~b}$ had the highest docking score of $144.368 \mathrm{kcal} / \mathrm{mol}$ indicating the better binding affinity against the target protein human DHFR (1KMS). Fig. 2 shows the threedimensional description of proposed binding mode and protein-ligand interactions of compound $5 \mathrm{~b}$ on active site residues of DHFR. From the docking analysis of compound $\mathbf{5 b}$ with human DHFR, it was recognized in view the formation of four hydrogen bonds enclosing the ligand with two interacting residues of the binding site. Nitrogen (N25) of compound $\mathbf{5 b}$ formed the hydrogen bond with TYR121 requiring the hydrogen atom of the amine group (A: TYR121: HN - 5b: N25) having a hydrogen bond distance of $1.908000 \AA$ and an oxygen atom of compound $5 b$ formed the hydrogen bond with VAL115 with a hydrogen bond distance of 2.292000 
Table 2: Cytotoxicity of synthesized compounds on MCF-7 cell line

\begin{tabular}{lll}
\hline S. No & Compound & IC $_{\mathbf{5 0}}$ values $(\boldsymbol{\mu g} / \mathbf{m l})$ \\
\hline 1 & $5 \mathrm{a}$ & 4.480 \\
2 & $5 \mathrm{~b}$ & 5.452 \\
3 & $5 \mathrm{c}$ & 21.62 \\
4 & $5 \mathrm{~d}$ & 39.3 \\
5 & $5 \mathrm{e}$ & 29.8 \\
6 & $5 \mathrm{f}$ & 4.215 \\
7 & $5 \mathrm{~g}$ & 26 \\
8 & $5 \mathrm{~h}$ & 2.253 \\
9 & $5 \mathrm{i}$ & 46.8 \\
10 & $5 \mathrm{j}$ & 7.67 \\
11 & $5 \mathrm{k}$ & 4.982 \\
12 & $5 \mathrm{l}$ & 34.1 \\
13 & $5 \mathrm{~m}$ & 27.3 \\
14 & $5 \mathrm{n}$ & 14.9 \\
15 & $5 \mathrm{o}$ & 19.8 \\
16 & $5 \mathrm{p}$ & 28.5 \\
17 & $5 \mathrm{q}$ & 6.671 \\
18 & $5 \mathrm{r}$ & 39.5 \\
19 & Standard & $15 \mathrm{nmol}$ \\
\hline
\end{tabular}

$\AA$. There was a single hydrogen bond formation between nitrogen (N25) atom of compound $5 \mathrm{~b}$ and a hydrogen atom (HH) of TYR121 (A: TYR121: $\mathrm{HH}$ - 5b: N25) with a hydrogen bond distance of $1.681000 \AA$. A hydrogen bond is formed when the hydrogen atom of VAL115b interacted with the oxygen atom of the compound 5b (A: VAL115: O-H45-5b) with a hydrogen bond distance of $2.465000 \AA$. Another hydrogen bond is formed between a hydrogen atom of TYR121 and the oxygen atom of the compound 5b (A: TYR121: H45 - 5b: OH) with a hydrogen bond distance of $2.292000 \AA$. Some non-bonded interactions are in the docking validation; the reference drug doxorubicin was docked into the binding site of human DHFR. The binding affinities of the synthetic compounds were compared and analyzed in reference to doxorubicin. An accurate analysis of the docking scores and interactions in comparison with that of reference reveals that the docked ligands were found to have similar binding poses with good and moderate scores, thus validating the adopted docking methodology. Herein, the bestscored compound 5b (144.368) exhibited relatively comparable binding score with appropriate confirmation that is very close to the reference doxorubicin (150.181 l), hence, showing better binding affinity.

\section{Cytotoxic activity}

The title compounds were evaluated for an antiproliferative activity through MTT ([3-(4, 5-dimethylthiazol-2-yl) -2,5-diphenyltetrazolium bromide]) based cytotoxic assay against MCF-7 breast cancer cell line with Taxol as standard reference and results were summarized in Table 2. All tested compounds have shown significant cytotoxic activity. Among all the tested compounds 5a, 5b, 5f, 5h, and 5k exhibited significant percentage inhibition of cell proliferation at an $\mathrm{IC}_{50}$ value of $4.480 \mu \mathrm{g} / \mathrm{ml}, 5.452 \mu \mathrm{g} / \mathrm{ml}, 4.215 \mu \mathrm{g} / \mathrm{ml}, 2.253 \mu \mathrm{g} / \mathrm{ml}$, and $4.982 \mu \mathrm{g} / \mathrm{ml}$.

\section{CONCLUSIONS}

The results of the present study demonstrated the synthesis of pyrazoline incorporated isoxazole derivatives and in silico evaluation for their efficacy as anticancer compounds through docking against hDHFR. Compound $\mathbf{5 b}$ is recognized as the most hopeful anticancer compound among the synthesized derivatives based on its highest docking score assuming the higher selective basis for hDHFR protein The synthesized compounds were evaluated the cytotoxic activity, compounds $\mathbf{5 a}, \mathbf{5 b}, \mathbf{5 f}$, $\mathbf{5 h}$, and $\mathbf{5 k}$ exhibited significant activity. Thus, the present study proposed the compounds $\mathbf{5 b}$ as the best effective inhibitor of human DHFR protein with significant anticancer activity and brings forth a new root line in designing inhibitors in the drug discovery process to treat cancer.

\section{ACKOWLEDGMENTS}

The authors thankful to G. Pulla Reddy College of Pharmacy and Department pharmacy, University College of Technology, Osmania
University, Hyderabad, India, for providing facilities. The authors are also thankful to Central University, Hyderabad, for providing spectral data.

\section{AUTHORS' CONTRIBUTIONS}

All the authors have contributed equally.

\section{CONFLICTS OF INTEREST}

We declare that there are no conflicts of interests.

\section{REFERENCES}

1. Hanahan D, Weinberg RA. Hallmarks of cancer: The next generation. Cell 2011;144:646-74.

2. Srivastava V, Kumar A, Mishra BN, Siddiqi MI. Molecular docking studies on DMDP derivatives as human DHFR inhibitors. Bioinformation 2008;3:180-8

3. Hagner N, Joerger M. Cancer chemotherapy: Targeting folic acid synthesis. Cancer Manag Res 2010;2:293-301.

4. Arooj M, Sakkiah S, Cao Gp, Lee KW. An innovative strategy for dual inhibitor design and its application in dual inhibition of human thymidylate synthase and dihydrofolate reductase enzymes. PLoS One 2013;8:e60470.

5. El-Feky SA, Abd El-Samii ZK, Osman NA, Lashine J, Kamel MA, Thabet HKh, et al. Synthesis, molecular docking and anti-inflammatory screening of novel quinoline incorporated pyrazole derivatives using the pfitzinger reaction II. Bioorg Chem 2015;58:104-16.

6. Rangaswamy J, Kumar HV, Harini ST, Naik N. Synthesis of benzofuran based 1,3,5-substituted pyrazole derivatives: As a new class of potent antioxidants and antimicrobials a novel accost to amend biocompatibility. Bioorg Med Chem Lett 2012;22:4773-7.

7. Michon V, du Penhoat CH, Tombret F, Gillardin JM, Lepage F, Berthon L. Preparation, structural analysis and anticonvulsant activity of 3-and 5-aminopyrazole N-benzoyl derivatives. Eur J Med Chem 1995;30:147-55.

8. Shi JB, Tang WJ, Qi XB, Li R, Liu XH. Novel pyrazole-5-carboxamide and pyrazole-pyrimidine derivatives: Synthesis and anticancer activity. Eur J Med Chem 2015;90:889-96.

9. Gurunanjappa P, Nagamallu R, Kariyappa AK. synthesis and antimicrobial activity of novel fused pyrazoles. Int J Pharm Pharm Sci 2014; 7:379-81.

10. Khunt RC, Khedkar VM, Chawda RS, Chauhan NA, Parikh AR, Coutinho EC, et al. Synthesis, antitubercular evaluation and 3D-QSAR study of N-phenyl-3-(4-fluorophenyl)-4-substituted pyrazole derivatives. Bioorg Med Chem Lett 2012;22:666-78.

11. el-Sabbagh OI, Baraka MM, Ibrahim SM, Pannecouque C, Andrei G, Snoeck R, et al. Synthesis and antiviral activity of new pyrazole and thiazole derivatives. Eur J Med Chem 2009;44:3746-53

12. Ansari A, Ali A, Asif M. Biologically active pyrazole derivatives. New J Chem 2017;41:16-41.

13. Rajput SS, Patel SN, Jadhav NB. Isoxazole a basic aromatic heterocycle: Synthesis, reactivity and biological activity. Int J Chem Tech Res 2015;8:297-17.

14. Zimecki M, Bąchor U, Mączyński M. Isoxazole derivatives as regulators of immune functions. Molecules 2018;23:2724.

15. Radhika T, Sravanthi S, Babu VH, Reddy BM. Synthesis, Biological evaluation and molecular docking studies of isoxazole synchronized quinazolinone derivatives. J Pharm Res 2017;11:895-2.

16. Loh B, Vozzolo L, Mok BJ, Lee CC, Fitzmaurice RJ, Caddick S, et al. Inhibition of HIV-1 replication by isoxazolidine and isoxazole sulfonamides. Chem Biol Drug Des 2010;75:461-74.

17. Panda SS, Chowdary PV, Jayashree BS. Synthesis, antiinflammatory and antibacterial activity of novel indolyl-isoxazoles. Indian J Pharm Sci 2009;71:684-7.

18. Durgamma S, Reddy PR, Padmavathi V, Padmaja A. Synthesis and antioxidant activity of amido-linked benzoxazolyl/benzothiazolyl/ benzimidazolyl-pyrazoles and isoxazoles. J Heterocycl Chem 2016;53:738-47.

19. Makarov VA, Riabova OB, Granik VG, Wutzler P, Schmidtke M. Novel [(biphenyloxy)propyl]isoxazole derivatives for inhibition of human rhinovirus 2 and coxsackievirus B3 replication. J Antimicrob Chemother 2005;55:483-8.

20. Basha SS, Divya K, Padmaja A, Padmavathi V. Synthesis and antimicrobial activity of thiazolyl pyrazoles and isoxazoles. Res Chem Int 2015;41:10067-83

21. Dabholkar VV, Ansari FY. Synthesis and characterization of selected fused isoxazole and pyrazole derivatives and their antimicrobial activity. J Serbian Chem Soc 2009;74:1219-28. 